\title{
Wozu räumliche Nähe in der urbanen Wissensökonomie? Eine geostatistische Analyse Wiener Forschungscluster
}

\author{
Robert Musil ${ }^{1}$ Jakob Eder ${ }^{1}$
}

Eingegangen: 8. Oktober 2015 / Angenommen: 4. November 2016 / Online publiziert: 23. November 2016

(c) Der/die Autor(en) 2016. Dieser Artikel ist eine Open-Access-Publikation.

Zusammenfassung Urbanisierung und Wissensorientierung der Ökonomie sind miteinander verknüpft und so werden Metropolregionen heute in der Regel als Innovationszentren betrachtet. Die Bedeutung des local buzz, also der spontane Austausch der Akteure, und das Vorhandensein von Erfahrungswissen (tacit knowledge), wurde durch neuere Studien allerdings in Frage gestellt. Es scheint, als wäre die Einbindung in globale Wissensnetzwerke (global pipelines) von größerer Bedeutung. Auf Basis einer räumlichstatistischen Auswertung von 1.363 Forschungseinrichtungen wird die Ausprägung des Wiener Forschungssektors (differenziert nach fünf Bereichen) ermittelt, wobei ausgeprägte räumliche Konzentrationen festgestellt werden. Des Weiteren zeigen Experteninterviews, dass sich für Wien die Relevanz von lokalen und/oder internationalen Kooperationen sowie die Gründe, die zu ,Wissensclustern“ führen, nicht verallgemeinern lassen. Räumliche Nähe wird teils durch historische Standorte vorgegeben und hat eine unterschiedliche Bedeutung für diverse Forschungssektoren. Räumliche Nähe ist folglich nicht als Widerspruch zur in der Buzz-pipeline-Debatte festgestellten Aufwertung der internationalen Kooperationen zu sehen. Sie übernimmt einerseits eine Kompensationsfunktion für Risiken, die durch internationale Kooperation entstehen, andererseits ist sie für gewisse Forschungsbereiche unumgänglich. Daraus ergeben sich folgende zwei Schlussfolgerungen: Die räumliche Innovationsforschung sollte sektorspezifische Konzentrationsmechanismen stärker berücksichtigen. Für die ur-

PD Dr. Robert Musil

robert.musil@oeaw.ac.at

1 Institut für Stadt- und Regionalforschung, Österreichische Akademie der Wissenschaften, Postgasse 7/4/2, 1010 Wien, Österreich bane Forschungspolitik zeigt sich die Notwendigkeit, die Standortanforderungen der jeweiligen Akteure stärker in ihre Konzepte und Strategien einzubeziehen.

Schlüsselwörter Urbane Ökonomie · Local buzz · Internationale Wissensnetzwerke · Cluster · Wien

\section{Is there a Need for Proximity in the Urban Knowledge Economy? A Geostatistical Analysis of Research Cluster in Vienna}

Abstract Urbanization and the knowledge economy are two concepts which are interlinked and therefore agglomerations are nowadays often considered as the centres of innovation. However, the importance of local buzz, in other words the spontaneous exchange of actors, and the existence of a tacit knowledge, was questioned in more recent empirical studies. There are indications that the access to global pipelines might be more important. Based on the analysis of 1,363 research facilities in Vienna (using spatial-statistical methods), the spatial patterns of Vienna's research sector (considering five sub-sectors) are determined and pronounced concentrations are found. In addition, expert interviews show that the relevance of local and/or international cooperation as well as the reasons which lead to "knowledge clusters" cannot be generalized. Spatial proximity is sometimes caused by historical locations and has different meanings for different research sectors. Hence, spatial proximity should not necessarily be seen as contradictory to the increasing importance of global pipelines, which is observed in recent studies. On the one hand, spatial proximity reduces risks, which are side effects of international cooperation. On the other hand, it is actually inevitable in some research sectors. This leads to the fol- 
lowing conclusions: First, the innovation research should take sector specific tendencies for concentrations more into account. Second, urban policy makers are advised to consider in detail the specific needs of the actors in question. In doing so, it is more likely, that research and development policies will succeed.

Keywords Urban economy · Local buzz · Global pipelines $\cdot$ Clusters $\cdot$ Vienna

\section{Einleitung}

Stadt und Wissensökonomie sind zwei eng verwobene Phänomene. Zahlreiche Theorien und Ansätze belegen das Innovationspotenzial der Metropolregionen und greifen dabei auf vielfältige Argumente wie die soziale und ökonomische Diversität (Jacobs 1969), die Standortpräferenzen der kreativen Klasse (Florida 2002), die hohe Dichte von lokalen Wissensnetzwerken (Lüthi/Thierstein/Bentlage 2010) oder die kreativen Milieus (Fromhold-Eisebith 1999) zurück. Die Möglichkeit des persönlichen Austauschs spielt dabei eine ganz besondere Rolle (Storper/Venables 2004), sowohl in produktionsnahen Clustern (Porter 1990) als auch in international tätigen Dienstleistungsunternehmen, die sich in den Global Cities konzentrieren (Sassen 1991). Das zugrundeliegende Argument, dass eine hohe Dichte und Diversität an Akteuren in einem eng begrenzten räumlichen Umfeld eine wichtige Voraussetzung für die Innovationsfähigkeit und Wissensproduktion darstellt, wurde allerdings von $\mathrm{Au}$ toren mit unterschiedlichen Argumenten in Frage gestellt.

Einerseits sind die Mechanismen unklar, wie die Wissensproduktion in lokalen Netzwerken erfolgt (Storper/ Venables 2004). Damit verbunden ist die Frage, wie das kodifizierte von nichtkodifiziertem Wissen abgegrenzt werden kann. Andererseits zeigen empirische Studien, dass nicht nur in der akademischen Praxis, sondern auch in der angewandten beziehungsweise unternehmerischen Wissensproduktion eher internationale Kooperationen als lokale Wissensnetzwerke eine zentrale Erfolgsvoraussetzung darstellen (Koo 2005; Bathelt 2008; Huber 2012). Führt diese, durch neue Kommunikationsmedien und virtuelle Formen räumlicher Nähe beschleunigte Entwicklung dazu, dass der Zusammenhang Stadt - Nähe/Dichte - Innovationsfähigkeit sukzessive erodiert und durch andere nichträumliche Formen von Nähe (Boschma 2005) substituiert wird?

$\mathrm{Zu}$ dieser Frage, zur Bedeutung räumlicher Nähe für die Wissensproduktion in urbanen Ökonomien, soll hier ein Beitrag geleistet werden. Dazu wird der Forschungsund Entwicklungssektor Wiens mit räumlich-statistischen sowie mit qualitativen Methoden analysiert. Wien ist in diesem Kontext als Fallbeispiel insofern von Interesse, weil es einerseits durch einen öffentlichen Forschungs- und Ent- wicklungssektor mit großen Forschungseinrichtungen geprägt ist, andererseits hat der private Forschungs- und Entwicklungssektor in den vergangenen Jahren vor allem im Bereich der kleinen Unternehmen beziehungsweise Startups ein starkes Wachstum verzeichnet. Damit rückt erstens die (räumliche) Verzahnung von öffentlichen und privaten Institutionen und zweitens die Frage nach der Bedeutung räumlicher Nähe für kleine Forschungs- und Entwicklungseinheiten in den Mittelpunkt.

Am Forschungsstandort Wien werden 1.363 forschungsorientierte Unternehmen und Institutionen auf der Basis der F\&E-Erhebung der Statistik Austria und der Wirtschaftsagentur Wien hinsichtlich des Ausmaßes der räumlichen Konzentration, ihrer Veränderung sowie den Tendenzen zur Clusterung analysiert. Anhand dieser Grundlage werden mittels Experteninterviews die Bedeutung der räumlichen Nähe sowie das Verhältnis zu globalen Wissens- und Kooperationsnetzwerken untersucht. In der empirischen Analyse wird sowohl zwischen unterschiedlichen Forschungsbereichen als auch nach öffentlichen und privaten Akteuren differenziert. Welche Rolle spielt räumliche Nähe für die Forschungsaktivität, welches Verhältnis lässt sich dabei zwischen dem öffentlichen und privaten Sektor feststellen?

\section{Forschungs- und Entwicklungsorientierung der urbanen Ökonomie - eine Frage der räumlichen Nähe?}

\subsection{Stadt und Wissensorientierung}

Die Produktion, Verbreitung und Adaption von Wissen und Kreativität sind zentrale Kennzeichen der modernen Wissensökonomie, die in steigendem Maß den urbanen Zentren zugeordnet sind. Um die Städte in diesem Sinne als, wissensbasierte Wachstumsmotoren' zu fördern, vollzog die europäische Stadtpolitik einen knowledge turn (van Winden 2010: 101). Sei es die Förderung der „kreativen Klasse“ in den Städten, die Entwicklung von Clustern oder die Selbstvermarktung als Wissensmetropole - Forschung und Entwicklung ist zu einem wichtigen Feld der aktuellen Kommunalpolitik geworden. Auch wenn sich hier ein gewisser Zeitgeist widerspiegelt, die Verzahnung von Stadt und wissensorientierter Ökonomie lässt sich in der historischen Rückschau zeigen. Der Aufstieg und das Wachstum der europäischen Metropolen waren seit der späten Industrialisierung (ab 1850, vgl. Hohenberg/Hollen-Lees 1985; Musil 2011) untrennbar mit der zunehmenden Innovations- und Wissensorientierung der Industrieproduktion verknüpft: Es waren die Städte, die aufgrund ihrer Größe diesen Branchen die notwendige Spezialisierung und Diversität boten.

Wie kann der Zusammenhang zwischen urbaner Ökonomie und Wissensorientierung erklärt werden? In der Lite- 
ratur lassen sich dazu unterschiedliche Erklärungsstränge ausmachen (vgl. Kujath 2005). Erstens sind Städte durch eine ausgeprägte ökonomische und soziale Diversität gekennzeichnet, die als zentrale Voraussetzung für ihr kreatives Potenzial angeführt wird (vgl. Jacobs 1969; Florida 2002; als Übersicht vgl. Storper/Venables 2004). Die hohe Dichte von horizontal und vertikal angrenzenden Branchen erleichtert die Entstehung von lokalen Wissensnetzwerken (Lüthi/Thierstein/Bentlage 2010) sowie kreativen Milieus (Fromhold-Eisebith 1999). Für Unternehmen entstehen dadurch formelle wie informelle Kontaktnetzwerke, die weniger Faktenwissen, als vielmehr Erfahrungen oder Kontextwissen verfügbar machen. Da dieses Wissen auf Face-toface-Kontakten basiert und hochgradig distanzsensibel ist, bilden sich in den betreffenden Städten Standortvorteile für dort niedergelassene Unternehmen.

Der zweite Erklärungsstrang greift weniger die Interaktionen und Lernprozesse innerhalb des urbanen Kontextes auf, sondern die Potenziale und Ressourcen, die die Städte $\mathrm{zu}$ Knoten in globalen Wissensnetzwerken machen. Hier ist der Global-City-Ansatz hervorzuheben, wonach Metropolen jene Orte sind, an denen das Wissen zum Management der Globalisierung geschaffen und bereitgestellt wird (Sassen 1991; Taylor 2004). Auch Simmie und Strambach (2006) heben im Kontext der urbanen Wissensproduktion hochgradig spezialisierte und räumlich konzentrierte Unternehmensdienstleister (,knowledge intensive business services“, KIBS) hervor, die aufgrund des interaktiven Wissenstransfers mit ihren Klienten (etwa multinationale Konzerne) und zwischen unterschiedlichen Branchen zu den zentralen ,Produzenten“ des urbanen Innovationspotenzials zählen.

Auch wenn die beiden Erklärungsstränge unterschiedliche Aspekte hervorheben (Milieus/lokale Netzwerke versus globale Wissensproduktion/Skaleneffekte), stellt der Aspekt der räumlichen Konzentration und damit der räumlichen Nähe ein zentrales Argument für die Verbindung von städtischen Räumen und der Wissensproduktion dar.

\subsection{Industriedistrikte, kreative Milieus und Cluster - Konzepte zur Erklärung regionaler Wissensproduktion}

Die Schlüsselfrage für urbane Ökonomien ist, welche Bedeutung räumliche Nähe für wissensorientiertes, ökonomisches Handeln hat. Die Argumente zur Erklärung der räumlichen Nähe sind nicht neu, sie gehen auf das Konzept der „Industriedistrikte“ von Alfred Marshall (1890) zurück. Hier sind die durch räumliche Nähe entstehenden Effizienzund Kostenvorteile für die Industrieproduktion ausschlaggebend, aber auch das Entstehen einer spezifischen, regionalen Unternehmenskultur (vgl. als Übersicht Palme/Musil 2012). Darüber hinaus hat in den 1990er-Jahren die fran- zösische GREMI ${ }^{1}$-Forschergruppe den Ansatz des „,kreativen Milieus“ entwickelt. Demnach ergibt sich das kreative und innovationsorientierte Potenzial aus der Konzentration unterschiedlicher sozialer Akteure: Unternehmen aus verschiedenen Branchen sowie Verwaltungs- und Bildungseinrichtungen. Diese bilden ein soziokulturelles Netzwerk in einem begrenzten lokalen Umfeld, in dem Lern- und Innovationsprozesse möglich sind (Fromhold-Eisebith 1999; Crevoisier 2001). Ein weiterer - und wohl auch der populärste - Ansatz, der die Vorteile der räumlichen Nähe für ökonomisches Handeln erklärt, ist die Clustertheorie von Michael Porter (1990; 2000). Ausgehend von berühmten Beispielen wie dem „Dritten Italien“ oder dem Silicon Valley wird ein Cluster als ein Netzwerk aus Unternehmen und öffentlichen sowie privaten Institutionen definiert, die durch arbeitsteilige und komplementäre Strukturen verflochten sind (vgl. als Übersicht Palme/Musil 2012; zur Kritik vgl. Martin/Sunley 2003; Kiese 2008).

Die Bedeutung eines Clusters - oder allgemeiner der räumlichen Nähe horizontal oder vertikal verbundener Unternehmen - ergibt sich neben Agglomerations- und Effizienzvorteilen vor allem aus dem lokalen Wissensaustausch, der wiederum die Quelle von neuem Wissen und Innovationen darstellt (Feldman/Audretsch 1999). Dabei spielt das tacit knowledge eine zentrale Rolle: Dieses entsteht durch kontinuierlichen, persönlichen Austausch und ist distanzsensibel, das heißt, die Qualität nimmt mit der Übertragungsdistanz ab. Daraus ergeben sich für Unternehmen Vorteile (neben den klassischen Spill-over-Effekten), in einem Cluster angesiedelt zu sein, um am dort vorhandenen Wissen partizipieren zu können. Begriffe wie local buzz (Bathelt/Malmberg/Maskell 2004) oder noise (Grabher 2002) umschreiben die auf Marshall zurückgehende industrial atmosphere, die räumliche Nähe, Face-to-face-Kontakte und das Vorhandensein eines tacit knowledge als zentralen Wettbewerbsvorteil begreift.

Das Konzept der Cluster zielt auf die Verbesserung der Wettbewerbsfähigkeit insbesondere in der Sachgüterproduktion (Porter 2000: 27) ab, was auch erklärt, warum die Argumente (Effizienzvorteile, gemeinsamer Arbeitsmarkt, Spezialisierungseffekte) jenen des wesentlich älteren Ansatzes der Industriedistrikte ähneln. Nicht zuletzt mit der zunehmenden Wissens- und Innovationsorientierung wurde auch zunehmend Kritik am Clusteransatz laut. Diese zielt einerseits auf die schwierige Abgrenzung und Erfassung von Clustern, andererseits auf die unklaren Mechanismen, die lokale Wissens- und Innovationsnetzwerke ausmachen (Storper/Venables 2004; Koo 2005; Huber 2012). Die zentrale Annahme der Bedeutung räumlicher Nähe für In-

\footnotetext{
${ }^{1}$ GREMI: Groupe de Recherche Européen sur les Milieux Innovateurs.
} 
novations- und Wissensproduktion wurde in der jüngeren Forschung auf unterschiedlichen Ebenen relativiert.

\subsection{Innovation ohne Nähe? Nationale und globale Wissensnetzwerke und die Substitution räumlicher Konzentration}

Empirische Befunde zeigen, dass für Unternehmen die Einbindung in internationale Wissensnetzwerke relevanter ist als lokale Kooperationen beziehungsweise der lokale Austausch (local buzz) (Giuliani 2007; Bathelt 2008; Moodysson 2008; Huber 2012). Aus diesem Grund wurde das Clusterkonzept dahingehend erweitert, dass neben dem local buzz auch globale Informationsnetzwerke - global pipelines - ausschlaggebend sind (Bathelt 2008: 88). Deren Funktion ist es vor allem, die Offenheit gegenüber externem Wissen zu sichern. Ausschlaggebend für den Erfolg eines Clusters ist demnach das richtige oder auch ausgewogene Verhältnis von local buzz und global pipelines.

Das Buzz-pipeline-Konzept ist nicht nur deshalb in die Kritik geraten, weil sich die empirischen Befunde des $l o$ cal buzz als dürftig herausgestellt haben, sondern auch, weil diese Dichotomie vereinfachend ist, da überregionale Wissensbeziehungen nicht ausreichend differenziert worden sind (vgl. Trippl/Tödtling/Lengauer 2009). Hier ist insbesondere der nationale Kontext hervorzuheben, da sich in nationalen Innovationssystemen sehr spezifische Formen institutioneller oder kultureller Nähe etabliert haben. Es zeigt sich insbesondere für den Wissenschaftsbereich, dass räumliche Nähe auf unterschiedliche Weise kompensiert werden kann: beispielsweise durch einen temporären local buzz, einem „Cluster auf Zeit“ in Form von Tagungen, Kongressen oder Forschungsaufenthalten (Trippl/Tödtling 2011). Ebenso führen neue Medien (wie etwa Facebook oder LinkedIn) dazu, dass soziale Kontakte und Vertrauen auch ohne räumliche Nähe geschaffen werden, also zu einem buzz without being there (Gertler 2008) führen.

Die Bedeutung räumlicher Nähe wird letztlich auch dadurch relativiert, dass unterschiedliche Formen von Nähe existieren (vgl. Boschma 2005; vgl. Tabelle 1), die fehlen- de räumliche Nähe kompensieren können. Dies gilt insbesondere für kognitive und organisatorische Nähe, die Wissensaustausch und Lernprozesse auch über größere räumliche Distanzen ermöglichen beziehungsweise in Kombination mit temporärer Nähe eine permanente Co-Lokation substituieren. Soziale Nähe drückt sich in Netzwerken aus, die per se a-räumlich sind. ,Vor Ort ${ }^{`}$ zu sein bedeutet also nicht, Teil eines lokalen sozialen Netzwerkes zu sein. Umgekehrt erleichtert räumliche Nähe die Schaffung von sozialer Nähe. Räumliche Nähe scheint weniger direkt, als vielmehr indirekt auf andere Näheformen zu wirken: ,geographical openness may enhance interactive learning more indirectly, most likely by realizing some distance with respect to the other dimension, such as cognitive distance" (Boschma 2005: 71).

\subsection{Branchenspezifische Formen (räumlicher) Wissensproduktion}

Die Bedeutung räumlicher Nähe hängt auch von der Art des Wissens - implizit/kodifiziert oder explizit/nichtkodifiziert (tacit knowledge) - ab, das in verschiedenen Branchen sehr unterschiedliche Bedeutung hat. Diesem Umstand wurde in dem Konzept der Wissensbasen Rechnung getragen, das drei Formen unterscheidet (Asheim/Coenen 2005; Asheim/ Coenen/Vang 2007):

- Die analytische Wissensbasis bringt Innovation durch die Schaffung neuen Wissens hervor und ist durch eine ausgeprägte Kooperation zwischen Universitäten, Forschungseinrichtungen und Forschungs- und Entwicklungsunternehmen gekennzeichnet. Ein local buzz für den Austausch des formalen Wissens spielt hier zwar eine relativ geringe Rolle, allerdings ist die Nähe zu Universitäten umso wichtiger für Start-ups, für den Zugang zu Infrastrukturen und Forscherteams. Beispielhaft sind moderne, wissenschaftsorientierte Forschungsbereiche wie Life Sciences oder Informations- und Kommunikationstechnologien.

Tab. 1 Typologie der Formen von nichträumlicher Nähe

\begin{tabular}{lll}
\hline \multirow{2}{*}{ Kognitive Nähe } & Charakteristika & Bezug zu räumlicher Nähe \\
erleichtert Kommunikations- und Lernprozesse, kann & Erfolgreiche geographische Cluster zeigen ein ausge- \\
aber auch zu einem lock-in führen & wogenes Maß an kognitiver Nähe \\
Soziale Nähe & $\begin{array}{l}\text { Unternehmenshierarchien oder Netzwerke, die den } \\
\text { Informations- und Wissensaustausch erleichtern }\end{array}$ & schafft Vertrauen über räumliche Distanz \\
& $\begin{array}{l}\text { Soziale Einbettung von Akteuren erleichtert Wissens- } \\
\text { austausch und Lernprozesse }\end{array}$ & $\begin{array}{l}\text { Soziale Nähe wird durch räumliche Nähe gefördert; } \\
\text { Agglomerationsfaktoren ermöglichen das richtige } \\
\text { Institutionelle Nähe }\end{array}$ \\
& $\begin{array}{l}\text { Formelle (Gesetze, Regeln) und informelle (Habi- } \\
\text { tus, kulturelle Normen) Institutionen schaffen stabile }\end{array}$ & Maß sozialer Nähe \\
& Beziehungen, die den Wissensaustausch fördern & oder international
\end{tabular}

Quelle: Eigene Darstellung, basiert auf Boschma (2005: 71) 
- In der synthetischen Wissensbasis dominiert implizites Wissen, da Innovationen eher durch die Adaptierung und Kombination vorhandenen Wissens geschaffen werden. Es steht ein anwendungsorientiertes Know-how im Vordergrund, das durch den Austausch mit Zulieferern oder Kunden entwickelt wird. Demnach spielen in diesen Branchen (etwa Anlagen- oder Maschinenbau) Faceto-face-Kontakte und räumliche Nähe der Akteure eine große Rolle.

- Die symbolische Wissensbasis greift ebenfalls auf überwiegend implizites Wissen zurück. Kreatives und personenbezogenes Wissen wird in soziale Netzwerke und Projekte eingebracht und kombiniert. Die Auseinandersetzung mit spezifischen Communities und (Jugend-)Kulturen spielt hier eine zentrale Rolle für die Wissensproduktion, die aus diesem Grund auf ein spezifisches, räumlich begrenztes urbanes Milieu konzentriert ist.

Die hier vorgestellten Typologien der Nähe sowie der unterschiedlichen Wissensbasen machen deutlich, dass die Branchen hinsichtlich der Bedeutung räumlicher Nähe für die Wissensproduktion sowie ihrer Standortanforderungen im Stadtraum beträchtlich variieren. Daraus ergeben sich für die urbane Ökonomie grundlegende Fragen: Ist für die Produktion von Wissen und Innovation räumliche Nähe überhaupt noch notwendig? Benötigen Akteure noch (zwingend) die Stadt mit ihren Diversitäts- und Spezialisierungsvorteilen, um spezifisches Wissen zu produzieren, wenn diese ohnehin in global pipelines eingebunden sind? Dies soll in diesem Beitrag anhand des Forschungs- und Entwicklungssektors in Wien empirisch untersucht und in diesem theoretischen Kontext diskutiert werden. Dazu lassen sich folgende Forschungsfragen formulieren:

- Wie verteilen sich öffentliche und private Forschungsund Entwicklungseinheiten, in welchem Ausmaß sind diese räumlich konzentriert?

- Wie wird das Verhältnis zwischen räumlicher Nähe und der Tendenz zur Internationalisierung bewertet?

- Lassen sich Unterschiede hinsichtlich der Bedeutung der räumlichen Nähe zwischen verschiedenen Forschungsund Entwicklungssektoren feststellen?

In dem empirischen Teil des Beitrages werden zuerst die Spezifika des Forschungsstandortes Wien herausgearbeitet, wobei das Verhältnis zwischen öffentlichen und privaten Akteuren im Vordergrund steht (Kapitel 3). Nach der Beschreibung der Methodik und Datengrundlage (Kapitel 4) werden die quantitativ-statistischen Ergebnisse zur Verteilung und Konzentration der Forschungs- und Entwicklungsakteure vorgestellt (Kapitel 5), anschließend die Experteninterviews zur Diskussion der Bedeutung räumlicher Nähe in der spezifischen Forschungspraxis (Kapitel 6). Der Bei- trag schließt mit einer Diskussion der Ergebnisse im Fazit (Kapitel 7).

\section{Struktur und Dynamik des Forschungs- und Entwicklungssektors in Wien}

Der Forschungssektor in Wien ist ganz deutlich von der Dominanz der Bundeshauptstadt in der österreichischen Hochschullandschaft geprägt. So sind beispielsweise mehr als die Hälfte $(53,9 \%)$ aller Studierenden in Österreich an einer Wiener Universität inskribiert (Musil/Eder 2013: 23). Dies drückt sich auch in der F\&E-Statistik aus: Im Vergleich zu den restlichen Bundesländern ist der Hochschulsektor wie auch der öffentliche Sektor in Wien deutlich überrepräsentiert. Der private Sektor macht in Wien 2011 gerade etwas mehr als die Hälfte der Wiener Vollzeitäquivalente (VZÄ) im Forschungs- und Entwicklungsbereich aus (51,7 \%), in den restlichen Bundesländern beträgt dieser Anteilswert hingegen 78,6 \%. 2011 wurden 34,7\% (2,8 Mrd. €) aller Forschungs- und Entwicklungsausgaben Österreichs in der Hauptstadt getätigt und gut ein Drittel aller F\&E-Beschäftigten des Landes $(33,9 \% ; 20.717$ VZ ̈̈) entfielen auf Wien (F\&E-Erhebung 2011, Statistik Austria).

In den vergangenen Jahren ist es zu einer beständigen Akzentuierung des Gegensatzes zwischen öffentlichen und privaten F\&E-Einheiten in Wien gekommen. Dies zeigt sich besonders deutlich bei den Forschungsausgaben. ${ }^{2}$ Während in Wien seit 2002 die Ausgaben des Hochschulsektors am stärksten $(+55 \%)$ und jene des privaten Sektors $(+27 \%)$ an geringsten wuchsen, ist die Situation in den restlichen Bundesländern genau umgekehrt: Dort sind die privaten F\&E-Ausgaben mit einer Zunahme um 111,4\% zwischen 2002 und 2011 mit Abstand am stärksten gewachsen (vgl. Abbildung 1) (Musil/Eder 2015: 36 ff.). Die Forschungslandschaft in Wien und dem restlichen Österreich scheint demnach in den vergangenen Jahren von unterschiedlichen Wachstumsmotoren geprägt worden zu sein.

Die Entwicklung des Forschungs- und Entwicklungssektors in Wien zeigt bei genauerer Betrachtung interessante Details. Seit 2007 sind in Österreich aufgrund politischer Entscheidungen die Globalbudgets der Universitäten um rund $25 \%$ erhöht worden, umgekehrt sind seit diesem Jahr die Ausgaben der privaten F\&E-Einheiten rückläufig (vgl. Musil/Eder 2015: 36). Damit bestätigt sich die Einschätzung von empirischen Studien, die festgestellt haben,

\footnotetext{
2 Als F\&E-Einheiten werden private und öffentliche Unternehmen bezeichnet, die in der Forschung oder experimentellen Entwicklung tätig sind und von der Statistik Austria im Rahmen der F\&EErhebung erfasst worden sind; vgl. http://www.statistik.at/web_de/ statistiken/energie_umwelt_innovation_mobilitaet/forschung_und_ innovation/f_und_e_in_allen_volkswirtschaftlichen_sektoren/index. html (16.10.2016).
} 
Abb. 1 Entwicklung der F\&EAusgaben des öffentlichen und des privaten Sektors sowie des Hochschulsektors in Wien (In$\operatorname{dex} 2002=100$ )

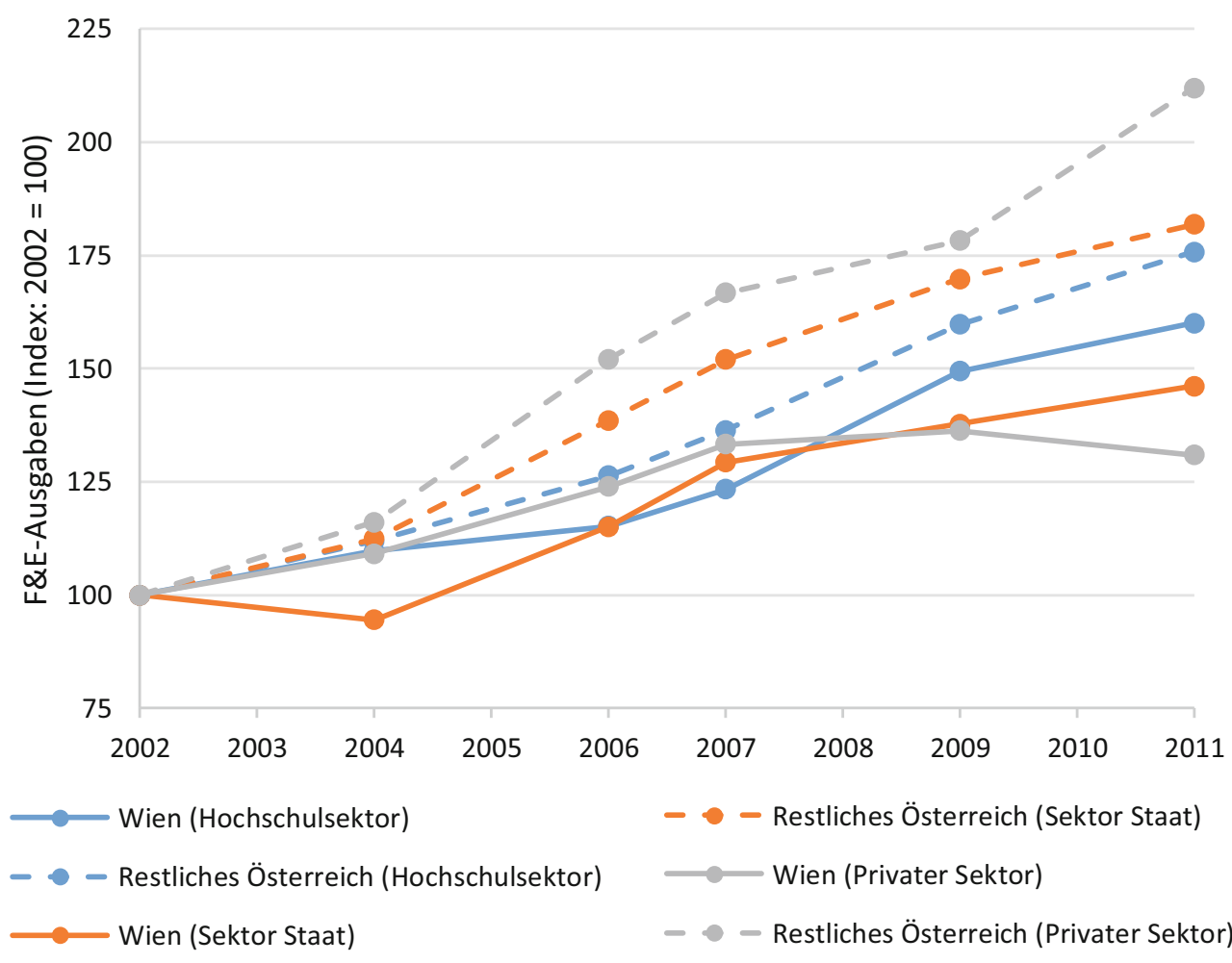

dass Unternehmen in Krisenperioden ${ }^{3}$ ihre Forschungs- und Entwicklungsausgaben reduzieren (Barlevy 2005; Cincera/ Cozza/Tübke et al. 2010). So haben beispielsweise einige große Pharmaunternehmen in den vergangenen Jahren ihre Forschungseinheiten in Wien aufgelassen oder reduziert, ebenso haben im Bereich der Informations- und Kommunikationstechnologien multinationale Konzerne innovationsorientierte Unternehmensbereiche vom Standort Wien abgezogen. ${ }^{4}$ Auffällig ist, dass parallel zu dieser Entwicklung (Rückgang der privaten F\&E-Ausgaben) die Zahl der privaten F\&E-Einheiten stark gestiegen ist: von 410 auf 791 zwischen 2002 und 2011 (Musil/Eder 2015: 36). Hintergrund ist ein Strukturwandel der privaten Forschungs- und Entwicklungslandschaft in Wien (vgl. Tabelle 2). Die Dominanz weniger großer F\&E-Einheiten ist zwar zu beiden Zeitpunkten enorm, allerdings hat die Gruppe der Großunternehmen (250 oder mehr Mitarbeiter) an Gewicht verloren. Deren Anteil an den wachsenden F\&E-Ausgaben reduzierte sich von 80,5 auf 69,7\% - eine Folge des Rückzuges

\footnotetext{
$3 \overline{\text { Hier handelte }}$ es sich um die durch die sogenannte Immobilienkrise ausgelöste „Finanzkrise“.

${ }^{4}$ Schließung des Novartis-Forschungsbüros in Wien: http://diepresse. $\mathrm{com} /$ home/wirtschaft/economist/349684/Wien_Novartis-schliesstForschungsburo (16.10.2016); Baxter bietet Mitarbeitern Wechsel von Wien nach Boston an: http://wirtschaftsblatt.at/home/boerse/ international/3878230/Baxter-bietet-Forschern-Jobwechsel-an_Bostonstatt-Wien (16.10.2016); IBM verlagert Osteuropa-Zentrale von Wien nach Prag und Dubai: http://derstandard.at/3364551/IBM-verlegtZentrale-fuer-Osteuropa-von-Wien-nach-Prag (16.10.2016).
}

der Großunternehmen bei gleichzeitiger Zunahme kleiner und mittlerer Unternehmen (Musil/Eder 2015: 38). In Wien scheint sich ein Trend zur Dekonzentration des privaten Forschungssektors zu vollziehen.

Die Einschätzung des Forschungsstandortes Wien fällt ambivalent aus: Aufgrund der starken Konzentration der Hochschullandschaft und der Erhöhung der Globalbudgets gewinnt die öffentlich finanzierte Forschung an Bedeutung. Eine Folge davon ist, dass Wien bei der experimentellen Forschung wie auch bei den Patentanmeldungen in Österreich - relativ gesehen - am hinteren Ende rangiert (Musil/ Eder 2015). Der private F\&E-Sektor ist hingegen von einem Strukturwandel, weg von wenig großen, hin zu einer Vielzahl an kleinen F\&E-Einheiten, geprägt. Dies ist ein Hinweis für die Belebung der innovationsorientierten Startup-Szene in Wien. Damit geht auch die Frage einher, ob und wie diese beiden Dynamiken - ein großer, dominierender Hochschulsektor und eine lebendige Start-up-Szene räumlich im Sinne eines local buzz zusammenhängen.

\section{Zur Methodik: Verteilung und Konzentration von Forschung und Entwicklung: Wie messen?}

Um die Forschungslandschaft in Wien hinsichtlich ihrer Verteilung und räumlichen Konzentration bewerten zu können, wurde ein Datensatz aufgebaut, der adressgenaue Informationen über 1.363 private und öffentliche Forschungseinrichtungen enthält. Aufgrund von Datenschutz- 
Tab. 2 Größenstruktur und Entwicklung des privaten Forschungssektors in Wien

\begin{tabular}{|c|c|c|c|c|c|c|}
\hline \multirow{3}{*}{$\begin{array}{l}\text { Unternehmensgröße } \\
\text { (Beschäftigte) }\end{array}$} & \multicolumn{3}{|l|}{2002} & \multicolumn{3}{|l|}{2011} \\
\hline & \multirow[t]{2}{*}{ F\&E-Einheiten } & \multicolumn{2}{|c|}{ Ausgaben für F\&E } & \multirow[t]{2}{*}{ F\&E-Einheiten } & \multicolumn{2}{|c|}{ Ausgaben für $F \& E$} \\
\hline & & in Mio. $€$ & in $\%$ & & in Mio. $€$ & in $\%$ \\
\hline 1 bis 9 & 99 & 15,5 & 1,3 & 349 & 58,5 & 3,9 \\
\hline 10 bis 49 & 114 & 56,6 & 4,9 & 202 & 139,0 & 9,2 \\
\hline 50 bis 249 & 91 & 152,9 & 13,3 & 133 & 261,1 & 17,3 \\
\hline 250 bis 999 & 45 & 260,8 & 22,6 & 60 & 281,8 & 18,7 \\
\hline 1.000 und mehr & 21 & 666,3 & 57,9 & 22 & 769,9 & 51,0 \\
\hline Gesamt & 370 & $1.152,3$ & 100,0 & 766 & $1.510,2$ & 100,0 \\
\hline
\end{tabular}

Quelle: F\&E-Erhebungen 2002 und 2011, Statistik Austria

auflagen waren für diese Einheiten nur die Zuordnung nach dem F\&E-Sektor (öffentlich, privat), nach dem Forschungsbereich, Adressdaten sowie allgemeine Merkmale (Name, Kontakt usw.) verfügbar. Die Grundinformationen stammen im Wesentlichen von der Statistik Austria sowie der Wirtschaftsagentur Wien und wurden durch eigene Recherchen ergänzt, insbesondere wurde die Größe der Forschungseinrichtungen geschätzt ${ }^{5}$ (vgl. Abbildung 2). Die Unterteilung in fünf Forschungsbereiche erfolgte auf der Basis der vorhandenen Daten mithilfe vorhandener Merkmale zur ÖNACE ${ }^{6}$-Zugehörigkeit für Unternehmen und der Arbeitsgebiete für öffentliche Forschungseinrichtungen ${ }^{7}$ sowie einer individuellen Online-Recherche. Ausreichend große Sektoren werden eigens ausgewiesen (Life Sciences, Informations- und Kommunikationstechnologie, Sachgüterproduktion), übrige Forschungseinrichtungen mussten zu den Human- beziehungsweise den Naturwissenschaften aggregiert werden, um die Mindestfallzahl für die weiteren Analysen nicht zu unterschreiten (vgl. Tabelle 3).

Aufgrund der Datenrestriktionen sind die räumliche Lage und die Sektorzugehörigkeit die wichtigsten Merkmale, die statistisch analysiert werden können. Damit wird das Methodenspektrum eingeschränkt und Verfahren der InputOutput-Analyse, wie sie etwa DeBresson und Hu (1999) oder Feser, Renski und Goldstein (2008) einsetzen, sind hier nicht anwendbar. Bergman und Feser (1999) schlagen zur Identifikation von Clustern neben Input-Output-Analysen zwei weitere Zugänge vor: einerseits statistische Verfahren, andererseits Experteninterviews, wobei letztere al-

\footnotetext{
5 Die Größe der F\&E-Einheiten wurde mittels Online-Recherche auf den Websites der Unternehmen ermittelt und in drei Größenklassen (1-9 Mitarbeiter, 10-49 Mitarbeiter, 50 Mitarbeiter und mehr) eingeteilt.

6 Österreichische Version der Nomenclature statistique des activités économiques dans la Communauté européenne: http://www.statistik. at/web_de/klassifikationen/klassifikationsmitteilung/beschreibung/ index.html (16.10.2016).

7 Arbeitsgebiete im Sinne der Österreichischen Systematik der Wissenschaftszweige: http://www.statistik.at/kdb/downloads/pdf/OEFOS_ 2012_Alphabetikum_A.pdf (16.10.2016).
}

lerdings einer gewissen Subjektivität unterliegen und damit die Identifikation neuer Cluster erschweren können.

Aus diesem Grund wird hier ein alternativer, mehrstufiger Zugang zur Identifikation und Analyse der Cluster gewählt. In einem ersten Schritt erfolgt eine deskriptive Analyse für fünf F\&E-Sektoren. Dazu werden Standardabweichungsellipsen (Standard Deviational Ellipse, SDE) berechnet, die näherungsweise zwei Drittel (bei einer Standardabweichung) der F\&E-Einheiten umschließen. Je kleiner die Fläche der Ellipse, umso größer ist das Ausmaß der räumlichen Konzentration. Des Weiteren dient eine Kerndichteschätzung (Kernel Density Estimation, KDE) dazu, die räumliche Konzentration zu visualisieren (vgl. Abbildung 3). Hier wird als Grundlage der existierenden Punktverteilung eine Dichte geschätzt, die als kontinuierliche Oberfläche dargestellt wird. Diese Interpolation ist sinnvoll, da erstens nicht sämtliche F\&E-Einheiten in der Datenbank enthalten sind (Unternehmen können sich dagegen entscheiden, im Katalog der Statistik Austria veröffentlicht $\mathrm{zu}$ werden) und zweitens am selben Standort liegende Einheiten stärker hervortreten.

Im zweiten Schritt wird - zur eigentlichen Identifikation der Cluster - ein Ansatz von Levine (2013) angewendet, der ursprünglich aus der Kriminalitätsforschung stammt und bislang nicht in der räumlichen Innovationsforschung eingesetzt wurde. Der Vorteil dieses Nearest Neighbor Hierarchical Clustering (NNHC) liegt darin, dass signifikante Cluster ausschließlich auf der Basis der räumlichen Lage (ohne Merkmalsdaten) ermittelt werden können und so auch die Ergebnisse nicht von einer vorangegangenen Klassifikation (etwa durch Statistikbehörden) beeinflusst werden. Es handelt sich folglich um eine Alternative zur Analyse von Input-Output- oder Informationsverflechtungen (vgl. Brandt/Hahn/Krätke et al. 2009), die angesichts der Datenlage nicht möglich ist. Überdies steht die Frage nach der räumlichen Nähe und deren Relevanz für Forschungs- und Entwicklungsaktivität im Mittelpunkt dieses Beitrages. Das Nearest Neighbor Hierarchical Clustering ermöglicht einen explorativen Zugang, mit dem - unabhängig von der Existenz offiziell eingerichteter und institutionell unterstützter 
Abb. 2 Räumliches Muster der F\&E-Einheiten in Wien

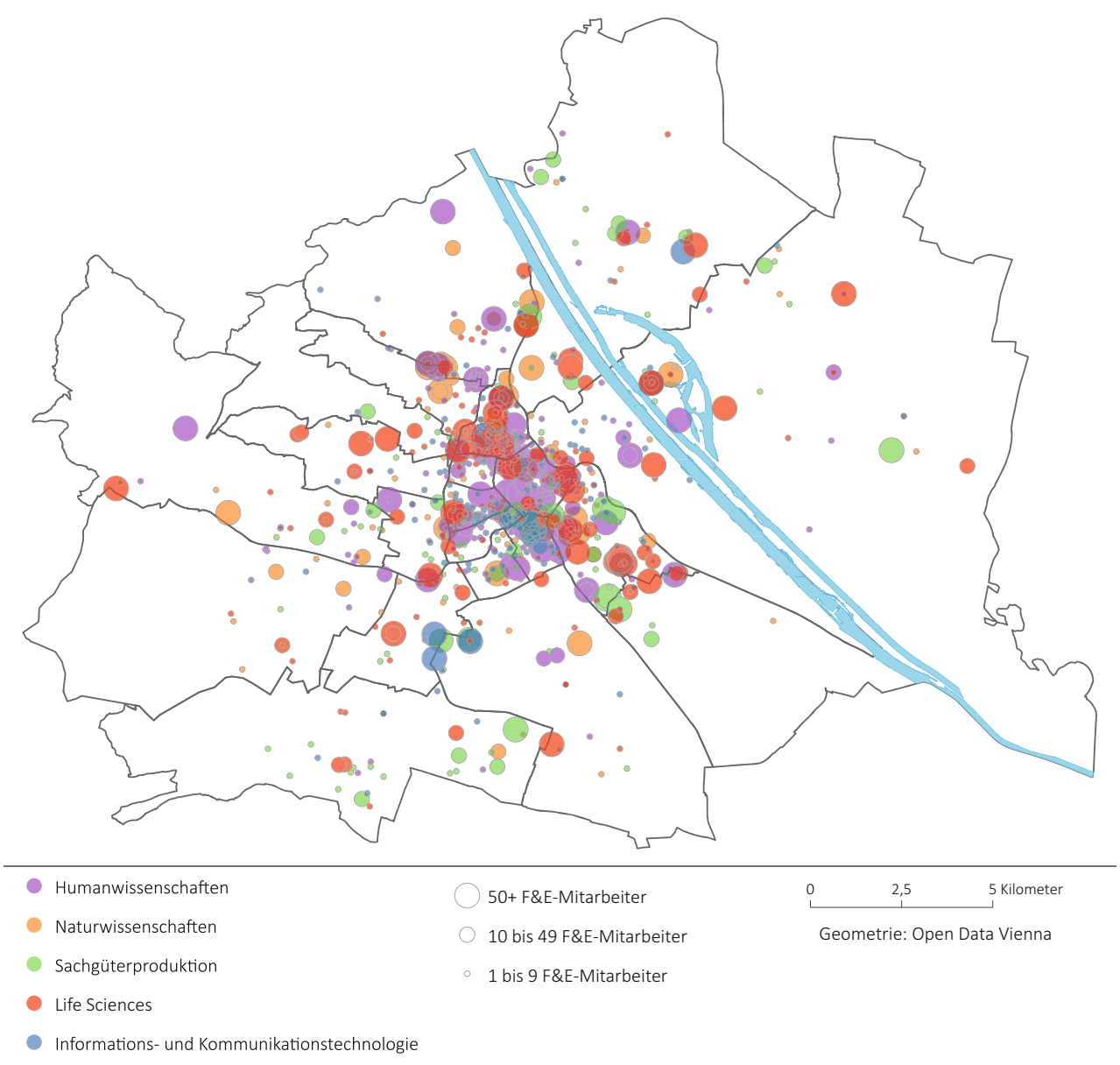

beziehungsweise ,institutionalisierter' Cluster - nach räumlichen Konzentrationstendenzen gesucht wird.

Im dritten Schritt werden die quantitativ identifizierten Clusterungen mittels Experteninterviews weiter analysiert, um die Gründe für Standortentscheidungen, die Bedeutung von Milieus und die Relevanz lokaler funktionaler Verflechtungen abschätzen zu können. Damit werden in diesem Beitrag quantitative und qualitative Ansätze miteinander verknüpft (Sui/DeLyser 2012). Der Vorteil dieses Zuganges liegt darin, dass die Frage nach der Lage und Existenz von Clustern mittels räumlich-statistischer Verfahren in einem Geographischen Informationssystem (GIS) beantwortet werden kann, die Gründe für eine etwaige Konzentration (die quantitativ nur sehr schwer fassbar sind) mittels qualitativer Interviews erhoben werden (Aitken/Kwan 2010).

\section{Räumliche Konzentration in der Wiener Forschungslandschaft - empirische Ergebnisse}

\subsection{Heterogene Konzentrationsmuster im Stadtraum}

Das Ausmaß der räumlichen Konzentration ${ }^{8}$ unterscheidet sich zwischen den fünf Sektoren beträchtlich, wobei die Bedeutung öffentlicher/akademischer Einrichtungen (insbesondere Universitäten) hier einen maßgeblichen Einfluss hat. Dies zeigt sich besonders im Forschungsbereich der Humanwissenschaften, bei denen es sich überwiegend um Universitäts- und öffentliche Forschungsinstitute handelt. Die historisch vorgegebene Standortstruktur der Wiener Universitäten ist dabei ausschlaggebend; diese sind überdies stark auf die Wiener Innenstadt konzentriert (vgl. Abbildung 3a). Nur die neue Wirtschaftsuniversität im 2. Bezirk und die Universität für Bodenkultur im 19. Bezirk reduzieren die überaus starke Konzentration auf das historische Stadtzentrum von Wien. Deutlich schwächer ist die

${ }^{8}$ Parameter SDE: basiert auf Standardabweichung $=1$ (schließt etwa $68 \%$ der Fälle ein); Parameter KDE: fixe Bandweite, Suchradius 750 m, basiert auf Quartic Kernel Funktion (vgl. Silverman 1986). 
Tab. 3 Struktur der F\&E-Datenbank

\begin{tabular}{|c|c|c|}
\hline F\&E-Bereich & Status & Fälle \\
\hline \multirow[t]{4}{*}{ Life Sciences } & Öffentlich & 180 \\
\hline & Privat & 152 \\
\hline & Gesamt & 332 \\
\hline & Anteil öffentlich & $54,2 \%$ \\
\hline \multirow{4}{*}{$\begin{array}{l}\text { Informations- und } \\
\text { Kommunikationstechnologie }\end{array}$} & Öffentlich & 26 \\
\hline & Privat & 154 \\
\hline & Gesamt & 180 \\
\hline & Anteil öffentlich & $14,4 \%$ \\
\hline \multirow[t]{4}{*}{ Sachgüterproduktion } & Öffentlich & 39 \\
\hline & Privat & 106 \\
\hline & Gesamt & 145 \\
\hline & Anteil öffentlich & $26,9 \%$ \\
\hline \multirow[t]{4}{*}{ Humanwissenschaften } & Öffentlich & 346 \\
\hline & Privat & 149 \\
\hline & Gesamt & 495 \\
\hline & Anteil öffentlich & $69,9 \%$ \\
\hline \multirow[t]{4}{*}{ Naturwissenschaften } & Öffentlich & 113 \\
\hline & Privat & 98 \\
\hline & Gesamt & 211 \\
\hline & Anteil öffentlich & $53,6 \%$ \\
\hline \multirow[t]{4}{*}{ Gesamt } & Öffentlich & 704 \\
\hline & Privat & 659 \\
\hline & Gesamt & 1.363 \\
\hline & Anteil öffentlich & $51,7 \%$ \\
\hline
\end{tabular}

Quelle: Projektdatenbank/eigene Erhebung (basierend auf F\&EErhebung 2011, Statistik Austria)

Konzentration im Bereich der technischen Wissenschaften und der Naturwissenschaften: Dieser Forschungsbereich ist ebenfalls stark von akademischen F\&E-Einheiten geprägt, allerdings fallen hier die peripheren Universitätsstandorte stärker ins Gewicht (vgl. Abbildung 3b).

Die geringste räumliche Konzentration weist hingegen der Forschungsbereich Sachgüterproduktion auf (vgl. Abbildung 3c). Zwar gibt es auch hier wieder mit der Technischen Universität (TU) Wien ein universitäres Zentrum, allerdings wird dieser Bereich von Produktionsunternehmen dominiert, die dispers über den Stadtraum verteilt sind. Aus der Perspektive der Clustertheorie mag die de facto nicht existierende Tendenz zur räumlichen Clusterung im Bereich der innovationsorientierten Sachgüterproduktion überraschen, dies ist möglicherweise auf den geringen Spezialisierungsgrad der Wiener Industrie zurückzuführen (vgl. Mayerhofer 2013). Eine ähnlich schwach ausgeprägte Konzentration findet sich im Life-Sciences-Bereich (vgl. SDE-Fläche, Abbildung 3d). Allerdings macht die kartographische Visualisierung deutlich, dass es hier vier Schwerpunkte gibt, an denen universitäre oder öffentliche Institutionen Gravitationskerne bilden: die Veterinärmedizinische Universität, der Standort Muthgasse (Universität für Bo- denkultur), die Medizin-Universität (Allgemeines Krankenhaus) sowie das Vienna Biocenter in St. Marx (3. Bezirk).

Am stärksten konzentriert ist der fünfte Forschungsbereich, der Sektor der Informations- und Kommunikationstechnologie, wobei die Technische Universität Wien den zentralen Gravitationskern bildet (vgl. Abbildung 3e). In dessen Umfeld konzentrieren sich zahlreiche forschungsorientierte Unternehmen der Informations- und Kommunikationstechnologie. Mit dem Informatik-Gebäude der Universität Wien existiert noch ein zweiter wichtiger Standort in Wien, um den sich jedoch fast ausschließlich öffentliche (universitäre) Einrichtungen gruppieren. Bemerkenswert an der Verteilung des Informations- und Kommunikationstechnologie-Sektors ist, dass trotz eines hohen Anteils $(85,5 \%)$ an privaten F\&E-Einheiten die Universitätsstandorte (vor allem die TU Wien) die räumliche Verteilung maßgeblich bestimmen und somit eine ausgeprägte Zentrumsorientierung dieses Forschungssektors besteht. Periphere Standorte spielen, von wenigen Ausnahmen abgesehen (Donaucity, Wienerberg City), nur eine untergeordnete Rolle.

In der Summe zeigen die fünf untersuchten Sektoren, dass die räumliche Konzentration, also die Nähe zu Akteuren im gleichen Forschungsbereich, sehr unterschiedlich ausgeprägt ist. Eine wichtige Rolle scheint dabei das Verhältnis zwischen öffentlichen und privaten F\&E-Einheiten zu spielen: Insbesondere in den ,gemischten“ Forschungsbereichen nehmen die Universitäten die Funktion von Gravitationskernen ein - besonders deutlich bei der Informations- und Kommunikationstechnologie, aber auch im Life-Sciences-Bereich. Damit stellt sich die Frage nach dem Ausmaß der Vernetzung von öffentlicher und privater Forschung. Ein weiterer Unterschied zwischen den Forschungsbereichen ist die Lage im Stadtraum. Während öffentliche F\&E-Einheiten in der Regel durch die Universitätsstandorte festgelegt sind, weisen die Unternehmen ein unterschiedliches Ausmaß an Zentrumsorientierung auf.

\subsection{Räumlich-statistische Identifikation von Clustern}

Um statistisch signifikante Cluster zu ermitteln, wurde eine Nearest Neighbor Hierarchical Clustering-Berechnung 9 durchgeführt (vgl. Kapitel 4). Levine (2013) merkt zwar an, dass die Methode ihre Schwächen hat, weil die Auswahl der Parameter einer gewissen Subjektivität unterliegt. Werden diese jedoch sorgfältig und im Hinblick auf die Fragestellung gewählt, sind die Ergebnisse valide.

Um aus dem Sample von 1.363 F\&E-Einheiten Ballungen zu identifizieren, wurden vier Kriterien formuliert, die auf jeden der fünf Forschungssektoren angewandt wurden.

\footnotetext{
${ }_{9}$ Parameter: Fläche Wiens $414,86 \mathrm{~km}^{2}$; Fixed Distance/festgelegte Distanz 500 m; Probability Value/Wahrscheinlichkeitswert 0,1; Minimum Points per Cluster: 10.
} 
Abb. 3 Kerndichte und Standardabweichungsellipse für diverse F\&E-Bereiche in Wien

a

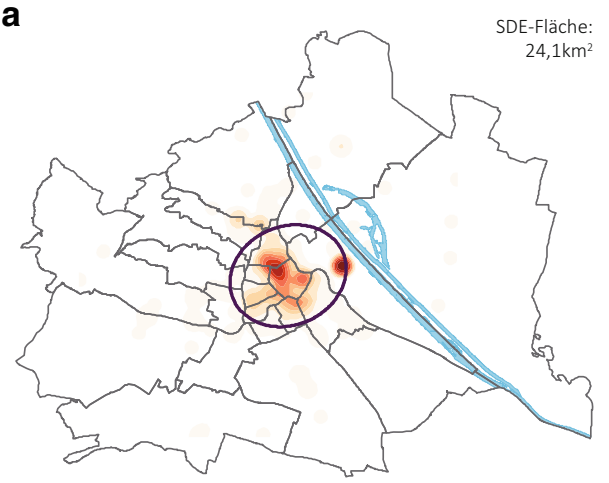

Kernel Density (geschätzte F\&E-Einheitne je $\mathrm{km}^{2}$ )*

\begin{tabular}{|c|c|c|}
\hline$>96.8$ & $>44.7$ to 59.9 & $\square>14.3$ to 23.5 \\
\hline$>77.5$ to 96.8 & | $>33.2$ to 44.7 & $\square>6.5$ to 14.3 \\
\hline$>59.9$ to 77.5 & $\square>23.5$ to 33.2 & $\square \geq 1.0$ to 6.5 \\
\hline
\end{tabular}

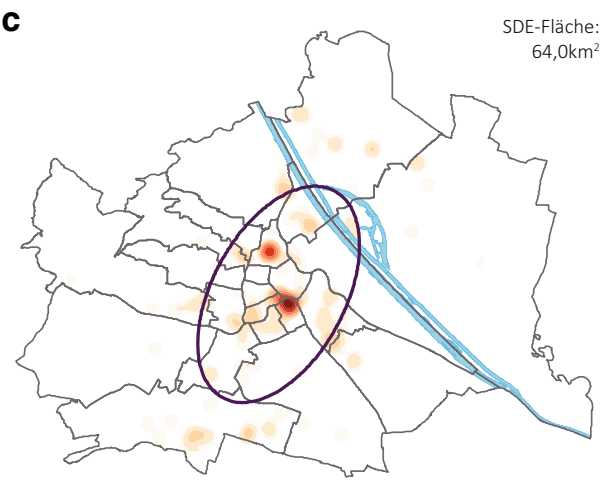

Kernel Density (geschätzte F\&E-Einheiten je km²)*
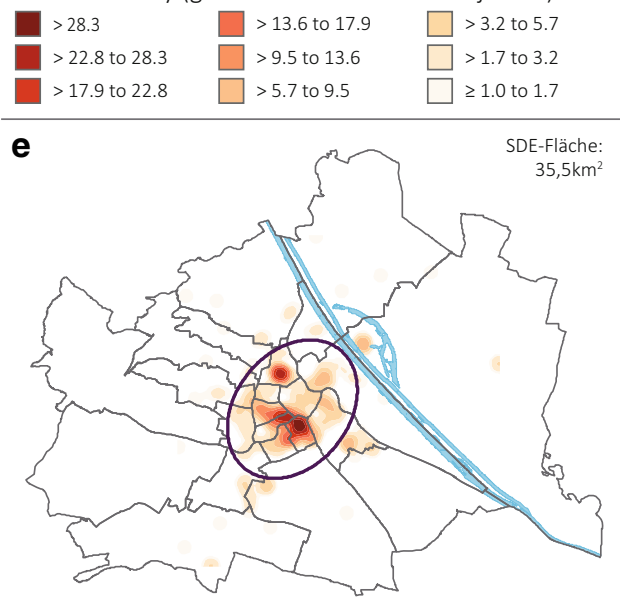

Kernel Density (geschätzte F\&E-Einheiten je $\mathrm{km}^{2}$ )*

\begin{tabular}{|c|c|c|}
\hline 22.4 & $>10.9$ to 14.4 & $\square>3.2$ to 5.1 \\
\hline$>18.1$ to 22.4 & $>7.7$ to 10.9 & $\square>1.7$ to \\
\hline $18, .1$ & $>5.1$ to 7.7 & $\geq 1.0$ to 1.7 \\
\hline
\end{tabular}

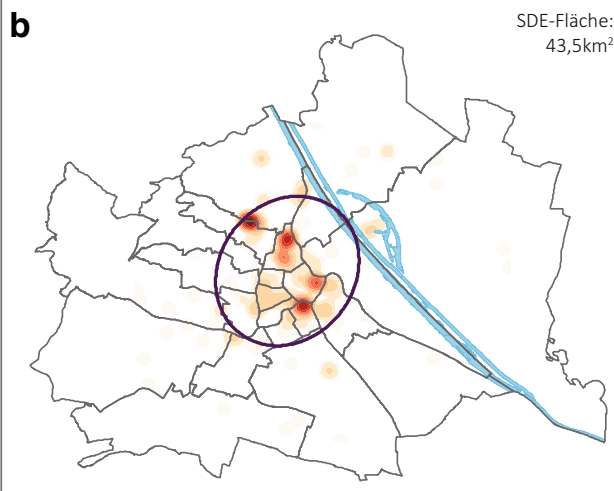

Kernel Density (geschätzte F\&E-Einheiten je $\mathrm{km}^{2}$ )*

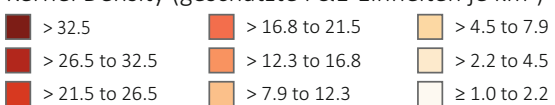

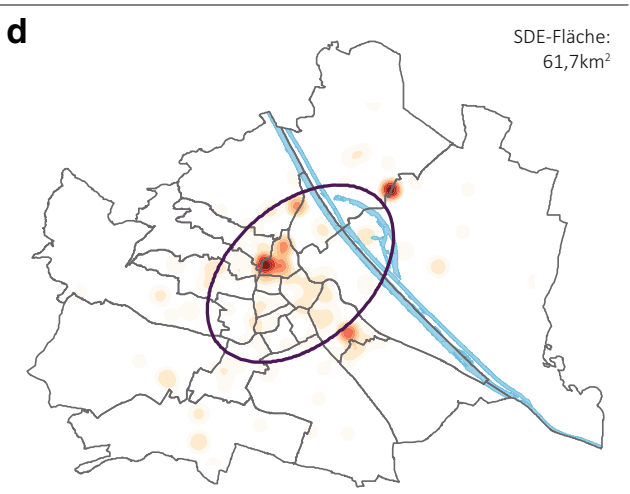

Kernel Density (geschätzte F\&E-Einheiten je $\mathrm{km}^{2}$ )*

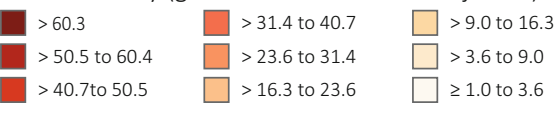

Durchschnittliche SDE-Fläche:
$44,1 \mathrm{~km}^{2}$

Standard Deviational Ellipse
(umfasst näherungsweise

$68 \%$ aller F\&E-Einheiten)

Donau

Wiener Gemeindebezirke

$0 \quad 510$ Kilometer

Geometrie: Open Data Vienna

*Werte unter 1.0 wurden von der Darstellung ausgeschlossen
Erstes Kriterium ist die räumliche Nähe zu anderen F\&EEinheiten, die mit einem Maximum von $500 \mathrm{~m}$ festgelegt wurde. Dies entspricht einer Gehzeit von etwa 10 Minuten und stellt die Obergrenze dar, die Personen bereit sind, in einem städtischen Umfeld zu Fuß zu gehen (Weidmann 1992:
35 ff.; Egartner/Fischer/Maißer et al. 2008: 32 f.; Stiewe/ Mühlhans/Bohnet et al. 2012: 609). Zweites Kriterium ist eine kritische Größe der Cluster (mindestens 10 F\&E-Einheiten, basierend auf einer Empfehlung in Levine (2013: 7.23)). Drittes Kriterium ist die Existenz von öffentlichen 
und privaten Einheiten (um reine Universitätsstandorte auszuschließen) und das vierte eine eindeutige fachliche Ausrichtung. Das heißt, der Cluster muss innerhalb eines der fünf Forschungsbereiche auftreten und nicht in der gesamten Stichprobe.

Folglich konnten fünf Clusterstandorte in Wien identifiziert werden, drei im Life-Sciences-Bereich (Allgemeines Krankenhaus, Muthgasse, Vienna Biocenter) sowie zwei im Bereich der Informations- und Kommunikationstechnologie (beide im Umfeld der TU Wien). In den anderen Forschungssektoren wurden durch Nearest Neighbor Hierarchical Clustering keine Konzentrationen ermittelt, die allen vier Kriterien entsprechen. Dieses Ergebnis deckt sich mit den oben beschriebenen Verteilungsmustern und zeigt, dass es gerade in den Forschungsbereichen Life Sciences und Informations- und Kommunikationstechnologie eine enge räumliche Verzahnung von öffentlichen und privaten F\&EEinheiten gibt.

Bemerkenswert ist allerdings der unterschiedliche Einfluss der kommunalen Forschungs- und Entwicklungspolitik auf diese beiden Standortmuster. Zwar sind beide Bereiche Schwerpunkte in der Forschungsförderung der Stadt Wien, jedoch mit einem entscheidenden Unterschied: Im Life-Sciences-Bereich wurde von der Stadt Wien über lange Jahre nicht nur Clusterpolitik, sondern in Abstimmung mit Universitäten, der Österreichischen Akademie der Wissenschaften sowie Unternehmen auch eine gezielte Standortpolitik betrieben (Tödtling/Trippl 2007; Tripp1/Tödtling 2007; für das Vienna Biocenter im Stadtteil St. Marx vgl. Wirth 2013). Für den Bereich der Informations- und Kommunikationstechnologie existierten (bis 2014) ebenfalls ein Clustermanagement sowie unterschiedliche Förderschienen und -initiativen, allerdings gab es keine gezielte Standortpolitik. Die starke räumliche Konzentration der F\&E-Einheiten im Bereich der Informations- und Kommunikationstechnologie im Umfeld der TU Wien beziehungsweise die zwei dort identifizierten Cluster lassen vielmehr darauf schließen, dass hier ein ,spontaner' Cluster entstanden ist, der von der (Förder-)Politik bislang nicht wahrgenommen worden ist.

Welche Rolle spielt also die räumliche Nähe sowie die Lage im Stadtraum für die unterschiedlichen Forschungsbereiche? Diese Frage soll in der Folge anhand von 14 leitfadengestützten Experteninterviews, die mit Vertretern aus dem Life-Science-Bereich (LS) sowie aus der Informations- und Kommunikationstechnologie (IKT) geführt worden sind, diskutiert werden. Dabei stammen sieben Interviewpartner aus öffentlichen und sieben aus privaten Forschungseinrichtungen. Mithilfe eines thematischen Rasters wurde anschließend analysiert, ob und welche Aussagen zu bestimmten Themenbereichen getätigt wurden und wie oft bestimmte Punkte zur Sprache kamen.

\section{Wozu räumliche Nähe? Erklärung und Bewertung der räumlichen Verteilungsmuster in den Bereichen Life Sciences und Informations- und Kommunikationstechnologie}

Ausgehend von der These einer zunehmenden Bedeutung der global pipelines für Forschungs- und Entwicklungsaktivitäten sowie den oben beschriebenen Konzentrationstendenzen in den untersuchten Forschungsbereichen, geht dieses Kapitel der Frage der Bedeutung räumlicher Nähe und lokaler Kooperationen für die Forschungspraxis nach. Diese Fragestellung ist für die beiden Branchen auch insofern interessant, als sie der analytischen Wissensbasis zugerechnet werden, für deren Wissensproduktion tendenziell überregionale Netzwerke und Kooperationen relevant sind. Dazu werden in der Folge die Ergebnisse von Experteninterviews diskutiert, die zwischen Oktober 2014 und Januar 2015 mit Forschungsleitern aus den Bereichen Life Sciences und Informations- und Kommunikationstechnologie durchgeführt wurden.

\subsection{Ambivalenz der global pipelines}

Die in der Literatur festgestellte Bedeutungszunahme globaler Kooperations- und Informationsnetzwerke (Bathelt 2008; Huber 2012) wurde in den Expertengesprächen überwiegend bestätigt, drei Argumente sind dafür ausschlaggebend. Erstens schränkt die zunehmende fachliche Spezialisierung im Wissenschaftsbetrieb die potenziellen Kooperationspartner stark ein und sie sind häufig weltweit nur an wenigen Standorten zu finden. Dies erschwert umgekehrt die Kooperationsmöglichkeit vor Ort, auch wenn ein gemeinsames lokales Milieu existiert. Andererseits gibt es dadurch keine lokale Konkurrenzsituation. Zweitens zwingt der kleine österreichische Binnenmarkt spezialisierte Nischenunternehmen zur Expansion, ebenso ist der Inlandsmarkt für klinische Studien zu klein und macht internationale Kooperationen notwendig. Drittens hat die Förderlandschaft einen wichtigen Einfluss auf das Kooperationsverhalten, da insbesondere bei EU-Programmen internationale Kooperationen vorausgesetzt werden.

„Wir haben einen sehr speziellen Biomarker entwickelt und da gibt es auf der Welt ungefähr [...] 500 Leute, die intensiv daran forschen.... Und deswegen sind wir natürlich auch global aktiv, dass wir unsere Umsätze steigern können“ (LS-1). „Das ist ganz einfach. Bei EU-Projekten kooperieren wir international, da muss man einen internationalen Partner haben" (IKT-1). „Wir schauen in welchem Land gibt es hohe Förderquoten. [...] Das heißt, das Förderinstrument dirigiert uns zu den verschiedenen Ländern“(IKT-2). 
Den genannten Argumenten, die eine Internationalisierung der Kooperationen und des Wissensaustauschs unabdingbar machen, stehen jedoch auch einschränkende Faktoren entgegen. Hier ist vor allem die zentrale Bedeutung des Vertrauens zu nennen, insbesondere im Bereich Life Sciences (vgl. auch Moodysson 2008; Aalbers 2010). Da der Erfolg von Kooperationen von der Zusammenarbeit mit den ,richtigen Personen " abhängt, ist diese immer personal driven und wird über lange Jahre, etwa durch Forschungsaufenthalte oder „temporäre Cluster“ (Maskell/Bathelt/Malmberg 2006; Trippl/Tödtling 2011) entwickelt. Des Weiteren wurden von den Interviewpartnern die hohen Kosten, sprachliche und kulturelle Barrieren als limitierende Faktoren der in Summe notwendigen - Internationalisierung angeführt. Die Aussagen bestätigen damit auch ältere Studien, die die Bedeutung der räumlichen Nähe für die Forschungspraxis im Life-Sciences-Sektor betonen (Tödtling/Trippl 2007).

„Keine Institutionen.[...] Also ich habe Kollegen [...], das sind Leute, das sind Fixpunkte in meinem stellaren System.[...] Personal driven, alles personal driven“ (LS-2). ,Es funktioniert mit moderner Kommunikation, also mit Skype und Co [...], dann, wenn die Leute einander gut kennen. Aber zuerst muss ich sie auf einen Ort bringen, dann Feste feiern - da lernen sie einander gut kennen, dann hat man die soziale Verbindung“(IKT-2). „Da hilft die räumliche Nähe auch bei Kollaborationen oder generell bei Interaktionen, weil man halt einfach die Barriere nicht hat. Wenn ich jetzt einen Kunden in Japan habe oder einen Partner in Japan, dann schaut das ein bisschen anders aus, oder Australien“ (LS-1).

\subsection{Vorteile der räumlichen Nähe}

In beiden Forschungsfeldern wurde von den Interviewpartnern räumliche Nähe als Vorteil für die Forschungspraxis genannt, jedoch teilweise mit unterschiedlichen Argumenten. So hoben Vertreter aus dem Bereich Informations- und Kommunikationstechnologie die Möglichkeit direkter sozialer Kontakte hervor, im formellen (Vorträge, Netzwerktreffen) wie auch im informellen (Mittagessen, Kaffeehausbesuch) Rahmen. Räumliche Nähe wird hier als fußläufige Distanz, etwa zu Restaurants oder der TU Wien, definiert. So werden im Bereich Informations- und Kommunikationstechnologie Kooperationspartner in unmittelbarer Gehdistanz bevorzugt. Mit räumlicher Nähe wird hier explizit auch ein beträchtliches $\mathrm{Ma} ß$ an Bequemlichkeit verbunden.

„Was wir schon gemacht haben mit dem Unternehmen hier, dass wir neben die TU gezogen sind. Es war für uns ganz klar, dass wir hier sein wollen und es gibt einen ganz engen Kontakt“ (IKT-2). „Wir fahren zum Beispiel sehr ungern, wir haben tatsächlich ein, zwei Firmenpartner, die so im 18. und 19. Bezirk sind, die öffentlich schlecht erreichbar sind. Das ist schon mühsam“ (IKT-3).

Die räumliche Nähe von Akteuren im Bereich Informations- und Kommunikationstechnologie im Umfeld der TU Wien hat auch zu einer Clusteridentität geführt, die den kooperativen Aspekt in den Vordergrund stellt. Dass dieser mit der Distanz abnimmt, hängt auch damit zusammen, dass es durch das gemeinsame Studium an dieser Universität eine zusätzliche Verbundenheit gibt, die durch diverse Aktivitäten der Informatikfakultät gezielt gefördert wird. Die Unternehmen suchen daher die Nähe zur TU Wien, waren in den Interviews zugleich über die beträchtliche räumliche Konzentration überrascht. Der Cluster existiert also, wird aber in seiner räumlichen Ausprägung nicht wahrgenommen. Die TU Wien ist ein wichtiger Angelpunkt des IKT-Clusters, sowohl auf der räumlich-materiellen wie auch auf der kulturell-milieuspezifischen Ebene.

„Nein, gar nicht, das ist eben interessant, weil ich das [Konkurrenz] viel mehr habe mit den Kollegen aus den Bundesländern, dort hat man aus irgendeinem Grund ein Konkurrenzdenken.[...] Vielleicht über die TU. Also es sind viele Absolventen, das sind schon wir, auch viele Gründungen sind von der TU, vielleicht hat man sich schon einmal wo gesehen" (IKT-2). „Nein, also ich bin überrascht. Das ist total interessant, weil es sich doch voll auf die TU Wien konzentriert" (IKT-4).

Im Bereich Life Sciences wird räumliche Nähe vor allem unter dem Effizienzaspekt gesehen, etwa um durchzuführende Messungen persönlich zu besprechen und direkt kontrollieren zu können. Inhaltliche Probleme und konkrete Fragen können leichter diskutiert und geklärt werden, wenn man über die Arbeitsgebiete der relevanten Personen Bescheid weiß und diese informell und kurzfristig persönlich treffen kann.

„Es ist immer am besten von Kollege zu Kollege, weil der weiß wirklich, was ich machen will und wirklich das, was ich mache oder gemacht habe und bereitstellen kann als Material“ (LS-3). „Also zum Beispiel habe ich ein Problem, dann kann ich wo anrufen und wir treffen uns vielleicht spontan in 20 Minuten, falls es geht. Das macht man und die physische Nähe hilft dann wirklich.[...] Man fährt nicht fünf Kilometer, um eine Messung zu machen, das geht einfach nicht" (LS-4).

Räumliche Nähe wird in diesem Kontext innerhalb desselben Gebäudes definiert. Zentrales Argument im Bereich Life Sciences stellt aus Kosten- und Effizienzgründen die 
Tab. 4 Internationalisierung und lokale Kooperationen: Argumente und Strategien der Risikominimierung in den Bereichen Life Sciences und Informations- und Kommunikationstechnik

\begin{tabular}{|c|c|c|c|}
\hline & Life Sciences & $\begin{array}{l}\text { Informations- und Kommuni- } \\
\text { kationstechnik }\end{array}$ & Beide \\
\hline $\begin{array}{l}\text { Argumente } \\
\text { für lokale Ko- } \\
\text { operation und } \\
\text { räumliche Nähe }\end{array}$ & $\begin{array}{l}\text { - Die gemeinsame Nutzung von Infra- } \\
\text { struktur ist zentral. } \\
\text { - Die Vorteile der räumlichen Nähe grei- } \\
\text { fen nur, wenn alle relevanten Akteure } \\
\text { und Instrumente/Einrichtungen so nah } \\
\text { wie möglich sind, am besten im selben } \\
\text { Gebäude. }\end{array}$ & $\begin{array}{l}\text { - Die räumliche Nähe zur TU } \\
\text { Wien schafft eine gewisse } \\
\text { Clusteridentität. } \\
\text { - Die Vorteile der räumlichen } \\
\text { Nähe greifen, wenn alle } \\
\text { Akteure fußläufig erreich- } \\
\text { bar sind. }\end{array}$ & $\begin{array}{l}\text { - Räumliche Nähe und Kooperation sind } \\
\text { vor allem in der Anfangsphase eines } \\
\text { Unternehmens bedeutend. } \\
\text { - Spontaner Austausch } \\
\text { - Bequemlichkeit } \\
\text { - Keine Konkurrenz aufgrund der hohen } \\
\text { Spezialisierung und der Nischenstrate- } \\
\text { gien. }\end{array}$ \\
\hline $\begin{array}{l}\text { Argumente für } \\
\text { internationale } \\
\text { Kooperation }\end{array}$ & & & $\begin{array}{l}\text { - Notwendig aufgrund (1) der hohen } \\
\text { Spezialisierung, (2) der globalen Ver- } \\
\text { marktung der Produkte und (3) interna- } \\
\text { tionaler Fördermittel (z. B. EU). }\end{array}$ \\
\hline $\begin{array}{l}\text { Strategien zur } \\
\text { Risikominimie- } \\
\text { rung }\end{array}$ & $\begin{array}{l}\text { - Nur persönliche Netzwerke werden für } \\
\text { Kooperationen genutzt, keine institutio- } \\
\text { nellen. } \\
\text { - Vertrauensbasis, persönliche Kontakte. }\end{array}$ & & $\begin{array}{l}\text { - Temporäre Nähe auf Konferenzen oder } \\
\text { durch Forschungsaufenthalte ist funda- } \\
\text { mental für Kooperationen. }\end{array}$ \\
\hline
\end{tabular}

Quelle: Experteninterviews/eigene Erhebung

gemeinsame Infrastruktur dar. Dies betrifft betriebswirtschaftliche Dienstleistungen (Buchhaltung, gemeinsames Bestellwesen) sowie auch die eigentliche Forschungstätigkeit, wie etwa die Verfügbarkeit von hochwertigen Mikroskopen, S3-Laboren oder Mouse-Facilities. ${ }^{10}$

„Wir haben diese Campus-Support-Facilities, wo Geräte angeschafft worden sind, die keine Institution alleine anschaffen hätte können. Das gibt uns natürlich wahnsinnig viel Aufwind und neue technische Möglichkeiten, die Etablierung neuer Technologien“ (LS-3).

Schließlich wurde in beiden Forschungsbereichen die Bedeutung von räumlicher Nähe für Start-ups sowie für Nachwuchsforscher hervorgehoben. Dies betrifft die Zugänglichkeit zu teuren Infrastrukturen (siehe oben), den spontanen Erfahrungsaustausch sowie praktische Gründe. Dabei gilt jedoch: Je etablierter, größer und somit eigenständiger ein Unternehmen ist, desto weniger ist es auf die räumliche Nähe angewiesen.

„Es hat sicher einen Vorteil, gerade in der Anfangsphase eines Unternehmens. Je größer das Unternehmen ist, desto unabhängiger ist es“ (LS-5). „Also wir haben sehr viele Projekte gehabt, gerade am Beginn, wo diese Nähe ausgenutzt worden ist, wo man gesagt hat: Wen kennt man in Wien?“ (IKT-2). „Auch dadurch, dass am Anfang vor allem Studenten an Bord waren, als Mitarbeiter oder als Freelancer, die konnten

10 S3-Labore: Labore mit höchster Sicherheitsstufe, ausgestattet mit biologischer Sicherheitsschleuse für gentechnische Forschung; MouseFacilities: Infrastrukturen für Mausmodellforschung, die von Universitäten häufig als Dienstleistungen für Tierversuche angeboten werden. dann zwischen ihren Vorlesungen ins Büro kommen, um Dinge abzuarbeiten, die in ihrem Kopf da entstanden sind" (IKT-5).

In beiden Forschungssektoren zeigt sich eine ambivalente Haltung zur Internationalisierung der Forschungsaktivitäten, wenngleich diese als unumgänglich betrachtet wird (vgl. Tabelle 4). Räumliche Nähe und lokale Kooperationen werden in beiden Bereichen als positiv bewertet, die Argumente unterscheiden sich aufgrund der jeweiligen Routinen und Prozesse im Forschungsalltag. Das gegenseitige Vertrauen ist für das Funktionieren von Forschungsprozessen essenziell, wobei im Bereich Life Sciences aufgrund der hohen Forschungskosten dieses noch höher einzuschätzen ist. Hier werden auch explizit Strategien zur Risikominimierung genannt, während im Bereich Informations- und Kommunikationsstrategie die räumliche Konzentration der Akteure im Umfeld der TU Wien als räumliche Strategie zur Risikominimierung verstanden werden kann.

\section{Fazit}

In dem Beitrag wurden quantitative und qualitative Verfahren angewandt, um die räumlichen Muster und Clusterbildungen des F\&E-Sektors in Wien zu analysieren. Die Ergebnisse zeigen, dass sich auch bei dieser eingeschränkten Datenlage mit Verfahren der räumlichen Statistik Clusterungen ermitteln lassen. Die Parametereinstellungen (fixed distance, points per cluster) des Nearest Neighbor Hierarchical Clustering basieren auf Empfehlungen in der Literatur, die natürlich einer gewissen Subjektivität unterliegen. Bislang existieren kaum Erfahrungen in der Anwendung dieser 
quantitativen Methode für die Innovations- und Clusterforschung. Es ist sicherlich noch Forschungsbedarf nötig, um diese Anwendung zu optimieren. Vor diesem Hintergrund zeigt sich umso mehr, wie wichtig es ist, die aus der Theorie abgeleiteten Thesen sowie die Ergebnisse quantitativer Analysen mittels qualitativer Interviews einer Plausibilitätsprüfung zu unterziehen.

Die so durchgeführte Analyse des Wiener Forschungssektors zeigt für den F\&E-Sektor insgesamt eine deutliche räumliche Konzentration. Zwischen den fünf untersuchten Forschungsbereichen haben sich jedoch beträchtliche Unterschiede feststellen lassen. In den Bereichen Humanwissenschaften und technische/Naturwissenschaften besteht eine ausgeprägte räumliche Konzentration, die durch die Universitätsstandorte, also durch öffentliche Akteure, historisch vorgegeben ist. Umgekehrt sind forschungsorientierte Einrichtungen beziehungsweise Unternehmen in der Sachgüterproduktion in der Peripherie des Stadtraumes dispers verteilt. Der Bereich Informations- und Kommunikationstechnologie weist eine ausgeprägte Konzentration auf, während der Bereich Life Sciences eine deutliche Mehrpoligkeit zeigt, die sich aus den universitären/öffentlichen Standortentscheidungen ergeben hat. Trotz der unterschiedlichen räumlichen Muster ist diesen beiden näher untersuchten Forschungsbereichen ein hoher Anteil an privaten Forschungseinrichtungen gemein, die um öffentlich finanzierte „Gravitationskerne“ angesiedelt sind. Besonders deutlich wird dies für den Bereich Informationsund Kommunikationstechnologie, da es im Umfeld der TU Wien nie eine gezielte Standortpolitik gegeben hat.

Aufgrund der Konzentration und der identifizierten Cluster in diesen beiden Forschungsbereichen stellt sich die Frage nach der Bedeutung der räumlichen Konzentration für die jeweilige Forschungspraxis. Diese ergibt sich im Bereich Informations- und Kommunikationstechnologie aus der Möglichkeit, von formellen und informellen Kontakten zu profitieren, die in fußläufiger Distanz möglich sind. Im Umfeld der TU Wien ist - trotz des Nichtvorhandenseins einer gezielten Clusterstrategie an diesem Standort eine Clusteridentität entstanden, die durch ein kooperatives Milieu geprägt ist. Hier scheint räumliche Nähe ganz klar dazu zu dienen, Risiken, die insbesondere mit der Internationalisierung einhergehen, zu kompensieren. Im Bereich Life Sciences stellt das Vertrauen ebenfalls eine gewisse Barriere der notwendigen Internationalisierung dar, wobei räumliche Nähe hier eine verhältnismäßig geringe Kompensationsfunktion einnimmt. Vielmehr wird diese durch den Nutzen gemeinsamer Infrastrukturen sowie die höhere Effizienz im Forschungsablauf erklärt.

Die Motive und Gründe für die räumliche Konzentration der hier untersuchten Forschungsbereiche sind sehr unterschiedlich. Dies ist nicht zuletzt deshalb bemerkenswert, da beide untersuchten Bereiche Teil der gleichen - der analy- tischen - Wissensbasis sind (Asheim/Coenen/Vang 2007). Für das Ausmaß der räumlichen Konzentration scheint weniger die Wissensbasis, als vielmehr branchenspezifische oder strukturelle Anforderungen ausschlaggebend zu sein. Daraus ergibt sich, dass in der räumlichen Innovationsforschung sektorspezifische Konzentrationsmechanismen stärker in den Vordergrund zu rücken sind. Dies unterstreicht des Weiteren die Notwendigkeit einer standortsensitiven Forschungs- und Entwicklungspolitik. In Abhängigkeit von den sektorspezifischen Anforderungen sind der Bedarf an räumlicher Nähe sowie die Frage, wo im Stadtraum Forschung und Entwicklung stattfinden soll, ein wichtiger Erfolgsfaktor.

\section{Datenquellen}

F\&E-Erhebungen, Statistik Austria 2002 bis 2011. Diverse Sonderauswertungen (01.08.2014).

Unternehmensdatenbank, LISA-Vienna, 2013

Unternehmensdatenbank, Wirtschaftsagentur Wien, 2013

Open access funding provided by University of Vienna.

Open Access Dieser Artikel wird unter der Creative Commons Namensnennung 4.0 International Lizenz (http://creativecommons.org/ licenses/by/4.0/deed.de) veröffentlicht, welche die Nutzung, Vervielfältigung, Bearbeitung, Verbreitung und Wiedergabe in jeglichem Medium und Format erlaubt, sofern Sie den/die ursprünglichen Autor(en) und die Quelle ordnungsgemäß nennen, einen Link zur Creative Commons Lizenz beifügen und angeben, ob Änderungen vorgenommen wurden.

\section{Literatur}

Aalbers, R. (2010): The role of contracts and trust in R\&D alliances in the Dutch biotech sector. In: Innovation: Management, Policy \& Practice 12, 3, 311-329.

Aitken, S.; Kwan, M.-P. (2010): GIS as Qualitative Research: Knowledge, Participatory Politics and Cartographies of Affect. In: DeLyser, D.; Herbert, S.; Aitken, S.; Crang, M.; McDowell, L. (Hrsg.): The SAGE Handbook of Qualitative Geography. Los Angeles, 287-304.

Asheim, B. T.; Coenen, L. (2005): Knowledge bases and regional innovation systems: Comparing Nordic clusters. In: Research Policy 34, 8, 1173-1190.

Asheim, B. T.; Coenen, L.; Vang, J. (2007): Face-to-face, buzz, and knowledge bases: sociospatial implications for learning, innovation, and innovation policy. In: Environment and Planning C 25, 5 , 655-670.

Barlevy, G. (2005): Why don't recessions encourage more R\&D spending? Chicago. = Chicago Fed Letter 220.

Bathelt, H. (2008): Knowledge-based clusters: regional multiplier models and the role of 'buzz' and 'pipelines'. In: Karlsson, C. (Hrsg.): Handbook of Research on Cluster Theory. Cheltenham, 78-92.

Bathelt, H.; Malmberg, A.; Maskell, P. (2004): Clusters and knowledge: local buzz, global pipelines and the process of knowledge creation. In: Progress in Human Geography 28, 1, 31-56. 
Bergman, E. M.; Feser, E. J. (1999): Industrial and Regional Clusters: Concepts and Comparative Applications. http://www.rri.wvu.edu/ webbook/bergman-feser/contents.htm (16.10.2016).

Boschma, R. (2005): Proximity and Innovation: A Critical Assessment. In: Regional Studies 39, 1, 61-74.

Brandt, A.; Hahn, C.; Krätke, S.; Kiese, M. (2009): Metropolitan regions in the knowledge economy: network analysis as a strategic information tool. In: Tijdschrift voor economische en sociale geografie 100, 2, 236-249.

Cincera, M.; Cozza, C.; Tübke, A.; Voigt, P. (2010): Doing R\&D or not (in a crisis), that is the question... Luxemburg. = IPTS Working Paper on Corporate R\&D and Innovation 12/2010.

Crevoisier, O. (2001): Der Ansatz des kreativen Milieus. Bestandsaufnahme und Forschungsperspektiven am Beispiel urbaner Milieus. In: Zeitschrift für Wirtschaftsgeographie 45, 3-4, 246-256.

DeBresson, C.; Hu, X. (1999): Identifying clusters of innovative activity: A new approach and a toolbox. In: OECD (Hrsg.): Boosting Innovation. The Cluster Approach. Paris, 27-59.

Egartner, S.; Fischer, T.; Maißer, M.; Meth, D.; Steinbichler, M.; Wächter, P.; Voigt, A. (2008): Stadt der kurzen Wege aus ökosozialer Sicht. Nahversorgung und Naherholung in Wien vor dem Hintergrund der Alterung. Wien.

Feldman, M. P.; Audretsch, D. B. (1999): Innovation in cities: Sciencebased diversity, specialization and localized competition. In: European Economic Review 43, 2, 409-429.

Feser, E.; Renski, H.; Goldstein, H. (2008): Clusters and Economic Development Outcomes. An Analysis of the Link between Clustering and Industry Growth. In: Economic Development Quarterly $22,4,324-344$

Florida, R. (2002): The Rise of the Creative Class. New York.

Fromhold-Eisebith, M. (1999): Das „kreative Milieu“ - nur theoretisches Konzept oder Instrument der Regionalentwicklung? In: Raumforschung und Raumordnung 57, 2-3, 168-175.

Gertler, M. (2008): Buzz without being there? Communities of Practice in Context. In: Ash, A.; Roberts, J. (Hrsg.): Community, Economic Creativity, and Organization. Oxford, 203-226.

Giuliani, E. (2007): The Selective Nature of Knowledge Networks in Clusters: Evidence from the Wine Industry. In: Journal of Economic Geography 7, 2, 139-168.

Grabher, G. (2002): Cool projects, boring institutions: temporary collaboration in social context. In: Regional Studies 36, 3, 205-214.

Hohenberg, P. M.; Hollen-Lees, L. (1985): The Making of Urban Europe 1000-1950. Cambridge, MA.

Huber, F. (2012): Do clusters really matter for innovation practices in information technology? Questioning the significance of technological knowledge spillovers. In: Journal of Economic Geography $12,1,107-126$

Jacobs, J. (1969): The Economy of Cities. New York.

Kiese, M. (2008): Stand und Perspektiven der regionalen Clusterforschung. In: Schätzl, L.; Kiese, M. (Hrsg.): Cluster und Regionalentwicklung. Theorie, Beratung und praktische Umsetzung. Dortmund, 9-50.

Koo, J. (2005): Knowledge-based Industry Clusters: Evidenced by Geographical Patterns of Patents in Manufacturing. In: Urban Studies 42, 9, 1487-1505.

Kujath, H. J. (2005): Die neue Rolle der Metropolregionen in der Wissensökonomie. In: Kujath, H. J. (Hrsg.): Knoten im Netz. Zur neuen Rolle der Metropolregionen in der Dienstleistungswirtschaft und Wissensökonomie. Münster, 23-63.

Levine, N. (2013): CrimeStat IV. A Spatial Statistics Program for the Analysis of Crime Incident Locations. Washington.

Lüthi, S.; Thierstein, A.; Bentlage, M. (2010): Standortverflechtungen in der Wissensökonomie. Über die Konnektivität der Metropolregionen Rhein-Main und Rhein-Ruhr. In: Geographische Rundschau 62, 11, 46-52.

Marshall, A. (1890): Principles of Economics. London.

Martin, R.; Sunley, P. (2003): Deconstructing clusters: chaotic concept or policy panacea? In: Journal of Economic Geography 3, 1, 5-35.
Maskell, P.; Bathelt, H.; Malmberg, A. (2006): Building global knowledge pipelines: The role of temporary clusters. In: European Planning Studies 14, 8, 997-1013.

Mayerhofer, P. (2013): Wiens Industrie in der wissensbasierten Stadtwirtschaft. Wandlungsprozesse, Wettbewerbsfähigkeit, industriepolitische Ansatzpunkte. Wien.

Moodysson, J. (2008): Principles and Practices of Knowledge Creation: On the Organization of "Buzz" and "Pipelines" in Life Science Communities. In: Economic Geography 84, 4, 449-469.

Musil, R. (2011): Die Metropolen Europas im Städtesystem des 20. Jahrhunderts. In: Matznetter, W.; Musil, R. (Hrsg.): Europa: Metropolen im Wandel. Wien, 15-38.

Musil, R.; Eder, J. (2013): Wien und seine Hochschulen. Regionale Wertschöpfungseffekte der Wiener Hochschulen. Wien. = ISRForschungsbericht 40.

Musil, R.; Eder, J. (2015): Local buzz in der Wiener Forschung. Wissensintensive Cluster zwischen lokale Einbettung und internationaler Orientierung. Wien. = ISR-Forschungsbericht 41.

Palme, G.; Musil, R. (2012): Wirtschaftsgeographie. Braunschweig.

Porter, M. (1990): The Competitive Advantage of Nations. New York.

Porter, M. (2000): Location, Competition, and Economic Development: Local Clusters in a Global Economy. In: Economic Development Quarterly 14, 1, 15-34.

Sassen, S. (1991): The global city. London, New York, Tokyo, Princeton.

Silverman, B. W. (1986): Density Estimation for Statistics and Data Analysis. London.

Simmie, J.; Strambach, S. (2006): The contribution of KIBS to innovation in cities: an evolutionary and institutional perspective. In: Journal of Knowledge Management 10, 5, 26-40.

Stiewe, M.; Mühlhans, H.; Bohnet, M.; Fricke, T.; Heller, S. (2012): Potenziale und Wirkungen standortbezogenen Mobilitätsmanagements. In: Schrenk, M.; Popvich, V.; Zeile, P.; Elisei, P. (Hrsg.): REAL CORP 2012. Re-Mixing the City. Towards Sustainability and Resilience? Schwechat, 605-614. = Proceedings REAL CORP 2012.

Storper, M.; Venables, A. J. (2004): Buzz: face-to-face contact and the urban economy. In: Journal of Economic Geography 4, 4, 351370.

Sui, D.; DeLyser, D. (2012): Crossing the qualitative-quantitative chasm I: Hybrid geographies, the spatial turn, and volunteered geographic information (VGI). In: Progress in Human Geography 36, 1, 111-124.

Taylor, P. (2004): World City Network. A global urban analysis. London, New York.

Tödtling, F.; Trippl, M. (2007): Knowledge Links in High-technology Industries: Markets, Networks or Milieu? The Case of the Vienna Biotechnology Cluster. In: International Journal of Entrepreneurship and Innovation Management 7, 2-5, 345-365.

Trippl, M.; Tödtling, F. (2007): Developing Biotechnology Clusters in Non-high Technology Regions. The Case of Austria. In: Industry and Innovation 14, 1, 47-67.

Trippl, M.; Tödtling, F. (2011): Regionale Innovationssysteme und Wissenstransfer im Spannungsfeld unterschiedlicher Näheformen. In: Ibert, O.; Kujath, H. J. (Hrsg.): Räume der Wissensarbeit. Zur Funktion von Nähe und Distanz in der Wissensökonomie. Wiesbaden, 155-169.

Trippl, M.; Tödtling, F.; Lengauer, L. (2009): Knowledge Sourcing beyond Buzz and Pipelines: Evidence from the Vienna Software Sector. In: Economic Geography 85, 4, 443-462.

van Winden, W. (2010): Knowledge and the European City. In: Tijdschrift voor economische en sociale geografie 101, 1, 100-106.

Weidmann, U. (1992): Transporttechnik der Fussgänger. Transporttechnische Eigenschaften des Fussgängerverkehrs. Literaturauswertung. Zürich. $=$ Schriftenreihe des IVT 90.

Wirth, M. (2013): Der Campus Vienna Biocenter. Entstehung, Entwicklung und Bedeutung für den Life Sciences-Standort Wien. Innsbruck. = Innovationsmuster in der österreichischen Wirtschaftsgeschichte 8 . 